\title{
(6) OPEN ACCESS \\ Parental presence on neonatal intensive care unit clinical bedside rounds: randomised trial and focus group discussion
}

\author{
Mohamed E Abdel-Latif, ${ }^{1,2}$ Danette Boswell, ${ }^{1}$ Margaret Broom, ${ }^{1}$ Judith Smith, ${ }^{1}$ \\ Deborah Davis ${ }^{3,4}$
}

\begin{abstract}
- Additional material is published online only. To view please visit the journal online (http://dx.doi.org/10.1136/ archdischild-2014-306724).

${ }^{1}$ Department of Neonatology, Centenary Hospital for Women and Children, Canberra Hospital, Woden, Australian Capital Territory, Australia ${ }^{2}$ School of Clinical Medicine, Australian National University, Woden, Australian Capital Territory, Australia

${ }^{3}$ ACT Health Directorate, Woden, Australian Capital Territory, Australia ${ }^{4}$ Faculty of Health, University of Canberra, Bruce, Australian Capital Territory, Australia
\end{abstract}

Correspondence to Professor Mohamed E AbdelLatif, Department of Neonatology, The Australian National University Medical School, Centenary Hospital for Women and Children, PO Box 11, Woden, ACT 2606 , Australia; Abdel-Latif. Mohamed@act.gov.au

Received 21 May 2014 Revised 23 December 2014 Accepted 26 January 2015 Published Online First 23 February 2015

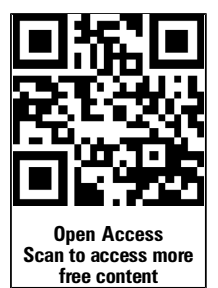

CrossMark

To cite: Abdel-Latif ME, Boswell D, Broom M, et al. Arch Dis Child Fetal Neonatal Ed 2015;100 F203-F209.

\section{ABSTRACT}

Background There are limited data to inform the choice between parental presence at clinical bedside rounds (PPCBR) and non-PPCBR in neonatal intensive care units (NICUs).

Methods We performed a single-centre, survey-based, crossed-over randomised trial involving parents of all infants who were admitted to NICU and anticipated to stay $>11$ days. Parents were randomly assigned using a computer-generated stratified block randomisation protocol to start with PPCBR or non-PPCBR and then crossed over to the other arm after a wash-out period. At the conclusion of each arm, parents completed the 'NICU Parental Stressor Scale' (a validated tool) and a satisfaction survey. After completion of the trial, we surveyed all healthcare providers who participated at least in one PPCBR rounding episode. We also offered all participating parents and healthcare providers the opportunity to partake in a focus group discussion regarding PPCBR.

Results A total of 72 parents were enrolled in this study, with 63 parents (87\%) partially or fully completing the trial. Of the parents who completed the trial, 95\% agreed that parents should be allowed to attend clinical bedside rounds. A total of 39 healthcare providers' surveys were returned and $35(90 \%)$ agreed that parents should be allowed to attend rounds. Nine healthcare providers and 8 parents participated in an interview or focus group, augmenting our understanding of the ways in which PPCBR was beneficial.

Conclusions Parents and healthcare providers strongly support PPCBR. NICUs should develop policies allowing PPCBR while mitigating the downsides and concerns of parents and healthcare providers such as decreased education opportunity and confidentiality concerns.

Trial registration number Australia and New Zealand Clinical Trials Register number, ACTRN12612000506897.

\section{BACKGROUND}

Current protocols and guidelines at some neonatal intensive care units (NICUs) do not allow parental presence at clinical bedside rounds (PPCBR) due to lack of high-quality evidence. Literature on PPCBR includes expert opinion, a handful of observational studies $^{1-7}$ and two randomised controlled trials $(\mathrm{RCT})^{89}$ conducted in general wards.

Foundations of patient and family centred care (FCC) are built on the tenets of respect, communication, participation, collaboration and inclusion of

\section{What is already known on this topic?}

Current practice on parental presence at clinical bedside rounds (PPCBR) varies between neonatal units. Issues of privacy and confidentiality are highlighted in previous studies of PPCBR as areas of main concern. There is a lack of quality research into families' views of ward rounds on neonatal units.

\section{What this study adds?}

PPCBR should be a core component of neonatal intensive care units (NICU) family centred care policy. PPCBR does not increase parents' stress as measured by the parental stressor scale for the hospitalised infant. NICUs should develop policies allowing PPCBR but minimising the negative effect such as decreased education opportunity and confidentiality concerns.

the family in all aspects of their baby's care. ${ }^{5}$ Davidson et $a l^{5}$ recommended that in order to provide FCC, parents need to be given the opportunity to participate in rounds so that they can ask questions and clarify information.

There is debate regarding the pros and cons of providing and facilitating PPCBR but the literature has yet to provide any strong evidence in support of a switch to PPCBR. It is up to individual neonatal units to make decision to allow PPCBR without a strong supporting evidence base, while also acknowledging that many people have strong opinions about the disadvantages of PPCBR. Disadvantages of PPCBR reported in the literature include the impact on resident teaching, the length of rounds and confidentiality for parents and the health professionals attending the rounds. ${ }^{2}{ }^{3}$

To the best of our knowledge, no randomised trial has evaluated PPCBR in NICU. Thus, we designed the PPCBR study, a survey-based crossover randomised trial, healthcare providers' (HCPs) survey and focus group discussion (FGD) to ascertain parents' and HCPs perspectives (communication and collaboration and confidentiality and privacy of information) and level of parental stress during both PPCBR and non-PPCBR interventions. 


\section{PATIENTS AND METHODS}

This study used a mixed-method approach including randomised trial, structured survey and FGD of parents and HCPs. The qualitative arm of the study aimed to add depth to the quantitative data by describing the participants' experiences with each model of care (PPCBR and non-PPCBR) along with perceived advantages, disadvantages and related issues.

\section{Ethics approval and trial registration}

Written informed consent from a parent or guardian for an infant fulfilling the eligibility criteria was obtained before participation in the trial. The trial was registered with the Australian New Zealand Clinical Trials Registry (ACTRN12612000506897) after the recruitment date. Sixty per cent of parents were recruited before the trial registration. There was no protocol amendment after the start of the trial.

\section{Trial design}

The trial design was a cross-over, single-centre, non-blinded randomised trial. The cross-over design was chosen for this study as it allowed the same family to experience both PPCBR and non-PPCBR interventions and thus be able to make an educated judgment about each model of care. Furthermore, it allowed each family to act as its own control, thus minimising the 'confounding' effect of factors that the researchers were not able to adequately adjust for, for example, sociodemographic factors, etc. Furthermore, this design increased the power of the trial compared with a parallel two-arm design.

\section{Trial randomisation procedure}

Parents were randomised to start with the PPCBR or non-PPCBR intervention using a computer-generated randomisation protocol. Parents were given a study number and were entered into a commercially available trial data management programme, Treonic (http://www.treonic.com). To ensure that equal numbers of parents of extremely premature infants were randomised to start with the PPCBR and non-PPCBR intervention, we stratified the randomisation by infant's gestation (gestation $\leq 30$ and $>30$ weeks) using randomisation block of four parents. Parents' allocation was revealed a day prior to the start of the trial.

\section{Trial blinding of study participants, healthcare providers} and researchers

Blinding of parents and HCPs and researchers was not possible because of the nature of the intervention.

\section{Parents' eligibility criteria}

Parent's eligibility for inclusion in the study depended on their infant's gestation at birth. Parents of infants born $\leq 30$-week gestation were eligible if it was thought that the infant would stay in NICU for $\geq 4$ weeks. Parents of infants born $>30$-week gestation were eligible if it was anticipated that the infant was going to stay in NICU for $\geq 11$ days. We allowed the first 2 days for not being included in the study as most mothers would be recovering from delivery/caesarean/anaesthesia and would not be able to participate in PPCBR in the first 2 days.

Parents of infants who were going to be transferred to another NICU or to their local hospital in $<11$ days after admission and parents who did not have good command of English were excluded from the study (we were not able to secure an interpreter to attend the rounds with families who did not have good command of English. Furthermore, this was thought to cause significant delays in clinical bedside rounds).

Eligible parents who consented for the study were given a handout summarising the PPCBR procedure, including an explanation of rounds. This handout informed parents about when rounds occurred and who would attend, gave details about upholding privacy and confidentiality during rounds, explained the role of rounds in teaching junior HCPs and medical students and emphasised that parents were welcome to ask questions and participate in the discussion about their infant. Although mothers' and fathers' experiences may differ, ${ }^{10}$ we chose to respect the couples' choice of attending the rounds and subsequent choice in completing the survey in consultation with each other or by any one of them.

\section{Trial protocol}

Once parents completed their initial randomly assigned arm (PPCBR or non-PPCBR), they were switched to the other arm after a washout period. The duration of each arm and washout period depended on the infant gestation. Infants who were $\leq 30$-week gestation, the duration of each arm and washout period was 1 week each (total study duration 3 weeks). Infants who were $>30$-week gestation it was 3 days (total study duration 9 days) as these infants are expected to be transferred to lower level units once in stable condition.

PPCBR involved attending the multi-disciplinary bedside clinical rounds. These rounds started between 8:30 and 9:00 and were attended by a neonatologist, neonatal fellow, day and night registrar, clinical nurse consultant, bedside nurse looking after the admitted infant, pharmacist, discharge planning nurse and medical students. Each baby's condition and test results were reviewed and a plan of care developed for the day under the supervision of the neonatologist.

At conclusion of the rounds parents were given an opportunity to ask questions about their baby's condition and management. When a lengthier discussion was required, a later meeting time was arranged to provide equal chance for other parents and maintain the continuous flow of the rounds. To preserve each family's privacy, parents were requested to leave the room when other babies were being discussed. After completing the PPCBR arm (ie, during non-PPCBR arm (routine care), washout period and after completing the trial), PPCBR was not allowed as the unit guidelines did not allow PPCBR in 2011-2012. Other than PPCBR, parents and their babies were treated according to the standard practice at Canberra Hospital NICU.

\section{Study outcome measures}

The primary trial outcome was Parental Stressor Scale (PSS) for hospitalised infants. The other trial outcome measures included parents' survey as explained below. To add depth to the trial, we designed a survey for HCPs and FGD for both parents and HCPs at end of the trial to describe their experiences with each model of care (PPCBR and non-PPCBR) along with perceived advantages, disadvantages and related issues.

\section{Parents' survey}

At the conclusion of each trial arm, parents completed two questionnaires: (1) satisfaction survey which was designed by the research team (see online supplementary appendix 1, section 1-5). This survey included questions regarding knowledge and understanding, communication and collaboration and privacy and confidentiality. (2) PSS which is a validated scale to measure how much stress parents have experienced as a result of their baby's illness and hospitalisation (see online 
supplementary appendix 1 , section 6). ${ }^{11} 12$ PSS includes a total of 22 questions covering 'parental role', 'infant appearance' and 'sight and sound' domains of parental stress in NICU.

\section{Healthcare providers' survey}

After completion of the trial, we surveyed all HCPs who at least participated in one PPCBR rounding episode (see online supplementary appendix 2 , section $1-4$ ).

\section{Focus group discussions}

All participating parents and HCPs were offered the opportunity to partake in a FGD regarding PPCBR. These FGDs were conducted by a single investigator (DD).

\section{Study location}

The study was conducted at the Department of Neonatology, Canberra Hospital, Australian Capital Territory (ACT), Australia. This unit is an urban 24-bedded tertiary NICU that serves ACT and the surrounding regional area of New South Wales, Australia. At the time of the study, the unit included one large open room, which was subdivided into NICU (eight beds), high dependency or intermediate (eight beds) and step-down or continuous care (eight beds) sections in addition to a single isolation room. At the time of the study, the routine care at Canberra Hospital (similar to many Australian NICUs) did not allow PPCBR in NICU. There was, however, an open-access visitation policy for parents except at morning rounds time (8:30 to $10: 00)$.

\section{Trial sample size calculation}

We used PSS of 3.5 (SD 0.67) from PSS validation study ${ }^{12}$ as a base for sample size calculation. A total of 60 patients were targeted to enter the two-arm cross-over trial. The probability is $80 \%$ that the trial will detect a treatment difference at a twosided 0.05 significance level, if the true difference between treatments is 0.350 units (10\%) and assuming that the within-patient SD of the response variable is 0.67 . We aimed to increase the sample size to 80 participants to account any loss to follow-up and discharge/transfer before completing the trial.

\section{Statistical analysis}

Statistical analyses of the trial data were performed using IBM SPSS Statistics (SPSS for windows; release V.20.0.0. SPSS: An IBM Company. Chicago, USA, 2012). Analyses were performed according to the intention-to-treat principle. The denominator that was used to calculate the percentage of each outcome was the number of parents/staff for whom that outcome was known. Data from PPCBR were compared with non-PPCBR. Continuous outcomes were analysed with the use of robust mixed-model-analysis (type III test of fixed effect) to obtain adjusted mean values with a 95\% CI. The results were adjusted for starting study arm, random effect and washout period. All analyses were prespecified. The level of statistical significance for all analyses was set at $\mathrm{p}<0.05$ using two-tailed comparisons. No adjustments were made for multiple comparisons. ${ }^{13}$

HCP survey was analysed as follows: data from Likert scales were reduced to nominal level by combining all 'agree and disagree' responses into two categories of 'agree/accept' and 'do not agree/reject'. $\chi^{2}$ and Fisher exact tests were used where appropriate.

The FGD were audio recorded and transcribed verbatim by a professional transcriber. Thematic analysis was undertaken following a qualitative descriptive approach. ${ }^{14} 15$
Because of the low-risk nature of the study, an interim safety and efficacy analysis and independent data and safety monitoring committee were not planned.

\section{RESULTS}

\section{Characteristics of the trial sample}

A total of 108 families were approached for recruitment with 72 families enrolled (figure 1). Thirty-seven parents were randomly assigned to start with PPCBR and 35 with non-PPCBR. Sixty-three families completed the study. The majority of the surveys were completed by mothers (table 1). Study participants attended $75 \%$ of allocated rounds.

\section{Parental support of PPCBR}

The majority of the parents supported PPCBR $(60 ; 95.2 \%)$, whereas only one (1.6\%) opposed and two (3.2\%) were undecided (figure 1).

\section{Parental satisfaction survey}

PPCBR had significantly higher adjusted mean (95\% CI) scores for some aspects of knowledge and understanding and communication and collaboration domain. Privacy and confidentiality domain was comparable between the two study groups (table 2).

\section{Parental Stressor Scale for hospitalised infant}

Adjusted analysis showed that the mean (95\% CI) PSS did not differ significantly between PPCBR (3.477 (3.262 to 3.692)) and non-PPCBR (3.638 (3.421 to 3.856)). When different domains of PSS were analysed individually, there was no significant between-group difference in the mean score (table 3). Further analysis stratified by the infant gestation (born $\leq 30$ or $>30$ weeks) showed similar results (table 3).

\section{Healthcare providers' survey}

The response rate for HCPs survey was $86.7 \%$ (39/45; 31 nurses and 8 medical officers). Thirty-five $(89.7 \%)$ of the HCPs supported PPCBR (table 3). HCP opinions on knowledge, communication, privacy and impact on their teaching during rounds are given in table 4 .

\section{FGD results}

Two FGDs, one with parents $(n=7)$ and one with HCPs $(n=9)$, were conducted. The HCPs' FGD included medical $(n=1)$, pharmacy $(n=1)$ and nursing staff $(n=7)$ with working experience between 1 and 20 years.

The parents' FGD included six mothers and two fathers aged between 28 and 42 years who had their babies spending between 3 and 9 weeks in NICU.

There was overall support for PPCBR from all participants; with one parent who was ambivalent about the amount of information shared at PPCBR, still supportive of parents having a choice regarding participation.

Two overarching themes, communication and philosophy, were apparent from FGD. These themes were the same for parents and HCP groups though the content varied between cohorts.

Parents and HCPs agreed that the depth and quality of information shared during PPCBR were beneficial and superior to the experience of non-PPCBR. Parents were frustrated with what they perceived as second hand' information relayed from neonatologists through nurses or junior medical staff that occurred during their non-PPCBR experiences. However, during PPCBR, they were comforted by knowing they had heard the information 'straight from the horse's mouth', 


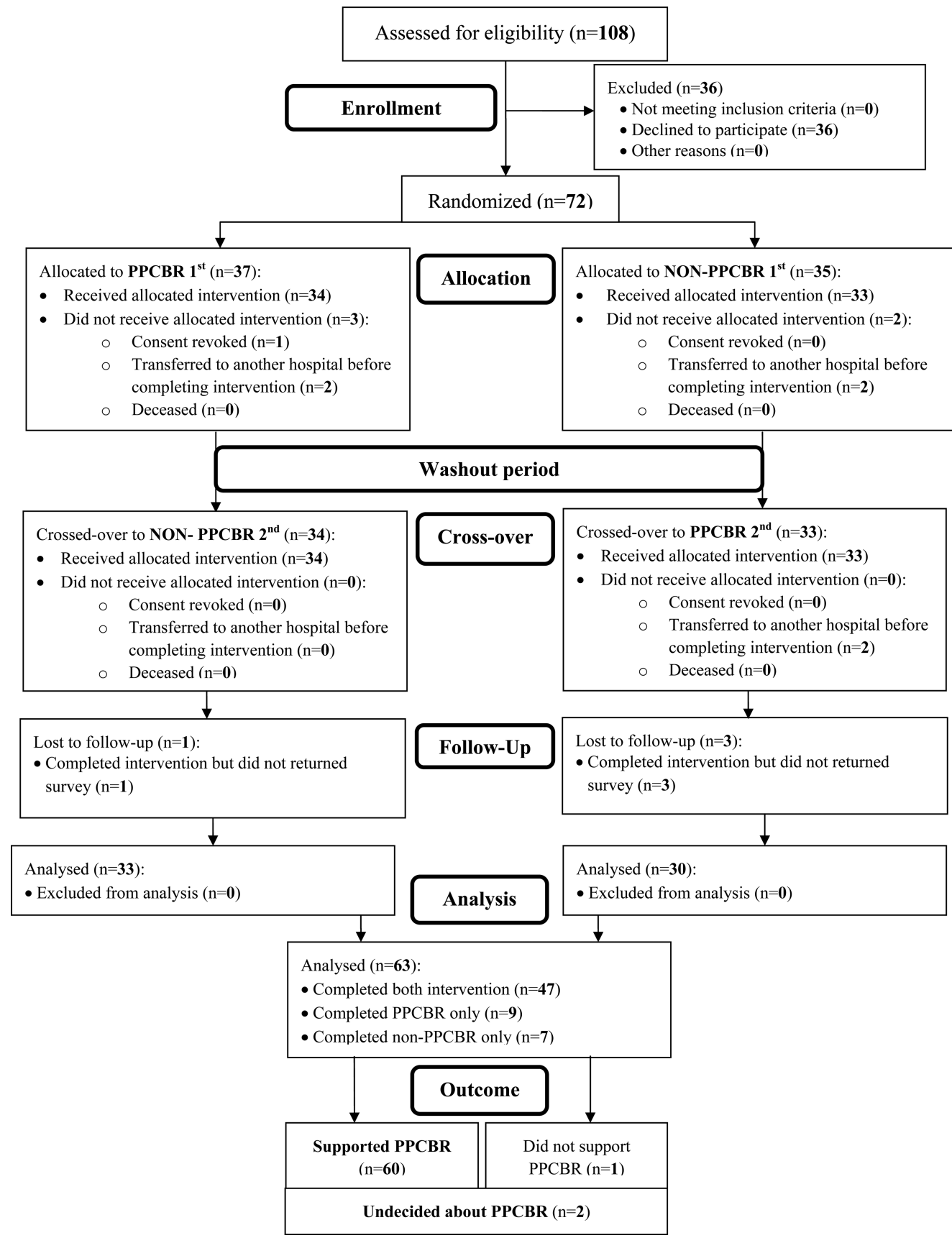

Figure 1 Flow diagram of study population from assessment for eligibility till completion of the cross-over randomised trial. PPCBR, parental presence at clinical bedside rounds.

something that HCPs also recognised. Parents also emphasised the opportunities that PPCBR afforded them in offering information that was useful to the clinical care of their baby. They described themselves as the one 'constant' in their babies' lives since HCPs were frequently changing.

While PPCBR had some disadvantages for HCPs including decreased opportunity for education, limiting frank discussion about the baby's condition and additional time involved in the round, the introduction of PPCBR brought practice into alignment with the unit's philosophy. It was not about philosophy for parents but an issue of 'rights'. They spoke passionately about their 'right' to be present and their 'right' to participate in the decisions concerning their baby. More importantly for parents, PPCBR provided them with an opportunity to 'be a parent'.

\section{DISCUSSION}

PPCBR is an important facet of FCC. PPCBR may improve patient care by improving parental understanding of a child's medical condition. Particularly in patients with complex or chronic conditions, this improved understanding may enhance parents' ability to optimally care for their children over the long term. ${ }^{2}$ The American College of Critical Care Medicine and the Society of Critical Care Medicine concurred that family participation in rounds is beneficial but acknowledged that there is lack of good-quality evidence especially in the acute care setting. $^{5}$

Our study is the first RCT in NICU in this area. It showed overwhelming parental and HCPs' support of PPCBR. We also showed overall better knowledge and understanding and communication and collaboration for the parental experience during 
Table 1 Characteristics of parents who completed PPCBR study

\begin{tabular}{lc}
\hline $\begin{array}{l}\text { Characteristics } \\
\text { Parent completed the survey }\end{array}$ & $\begin{array}{c}\text { Number (\%) or } \\
\text { median (IQR) ( } \mathbf{n = 6 3 )}\end{array}$ \\
Mother & $53(84)$ \\
Father & $10(16)$ \\
Completed the survey in consultation with the other partner/spouse \\
Yes & $26(41)$ \\
No & $37(59)$ \\
Age of parent completed the survey & $30.6(20-41)$ \\
Parent completing the survey highest level of & $43(68)$ \\
education attained is diploma, bachelor or higher & \\
Partner/spouse's highest level of education attained is & $33(52)$ \\
diploma, bachelor or higher & \\
Language other English spoken at home & $4(6)$ \\
Another child admitted to NICU before & $3(5)$ \\
Rounds actually attended out of allocated rounds (\%) & $75(15-100)$ \\
Infant gestation, weeks & $31(25-42)$ \\
Infant birth weight, g & $1696(420-4515)$ \\
\hline NICU, neonatal intensive care units; PPCBR, parental presence at clinical bedside \\
rounds.
\end{tabular}

PPCBR. Infant and family privacy and confidentiality were upheld and PSS was not increased during PPCBR.

Previous studies conducted in paediatric intensive care units supported PPCBR and found that there was better exchange of information between clinicians and families, increased parental empowerment, transparency of thought and data and satisfaction with their child's care as the positive aspects of PPCBR. ${ }^{2} 616{ }^{17}$ Furthermore, parents who participate in rounds may be more in tune with plans for the day and may potentially help expedite interventions or discharge planning. ${ }^{3}$ Bramwell and Weindling ${ }^{4}$ conducted a qualitative study, which showed that families have raised concerns regarding confidentiality, with parents admitting that they overheard reports on other babies.
However, our study did not show similar results, probably because parents were requested to leave the room when other infants were being discussed.

HCPs' concerns with PPCBR are also reported in the literature, including limiting clinicians' discussions of potentially sensitive topics, a likely negative impact on junior HCPs and resident teaching, possible erosion of parental confidence in junior HCPs, crowding, increased length of rounds and confidentiality concerns for patients and HCPs. ${ }^{2} 3516$ Our HCPs' survey reported similar concerns. In contrast, Phipps et al ${ }^{6}$ did not demonstrate a negative impact of PPCBR on the medical discussion during rounds. Cameron et $a l^{2}$ suggested that a hybrid system in which parents are included for a portion of rounds and some time is set aside for medical team discussion is a possible solution for HCPs' concerns.

This study is not without limitations. Blinding of parents and HCPs was not possible because of the nature of the intervention. It is acknowledged that incomplete round attendance rate may have had an effect on these results. However, this study's rate of $75 \%$ is much higher than in other studies (cf. 37\% in Cameron $e t a l^{2}$ ). The Likert scale used in our survey is also known to have an acquiescence and nay-saying bias (the inclination to agree or disagree) with a statement when there is doubt. ${ }^{18}$ However, in an attempt to minimise this bias an equal number of questions which both support and disagree with PPCBR were used. Social desirability bias ${ }^{19}$ may also have had an effect on the survey. However, this bias is minimised by the anonymity of the respondent. Furthermore, being a single centre, the study is prone for selection bias. ${ }^{20}$ Whether the 'equivalence' in PSS between PPCBR and non-PPCBR shown in our study is sustained (Hawthorne effect) is difficult to say and will require further research. Furthermore, our small sample size means that our study may not be sufficiently powered to detect a difference between the groups (type II error). However, this is unlikely given that our CIs are very tight.

In conclusion, we found that inclusion of parents on bedside medical rounds as a part of FCC is strongly supported by parents and HCPs. Parents should be routinely invited to

Table 2 Opinion of parents who completed PPCBR study on knowledge, communication and privacy and their Parental Stressor Scale for hospitalised infant

\begin{tabular}{|c|c|c|c|c|}
\hline & \multirow{2}{*}{$\begin{array}{l}\text { Number of } \\
\text { responses } \\
\text { analysed } \\
(n=126)^{*}\end{array}$} & \multicolumn{2}{|c|}{ Adjusted mean $(95 \% \mathrm{Cl})$} & \multirow[b]{2}{*}{ p Value } \\
\hline & & PPCBR & Non-PPCBR & \\
\hline \multicolumn{5}{|l|}{ Knowledge and understanding } \\
\hline I have received adequate information about my baby's condition and management & 105 & 4.321 (4.092 to 4.551$)$ & 3.947 (3.712 to 4.182 ) & 0.03 \\
\hline The healthcare team explained things thoroughly using easy to understand language & 105 & 4.325 (4.114 to 4.537$)$ & $4.230(4.013$ to 4.446$)$ & 0.49 \\
\hline The information I have received has been appropriate and timely & 104 & 4.057 (3.845 to 4.270$)$ & 4.357 (4.119 to 4.595$)$ & 0.13 \\
\hline \multicolumn{5}{|l|}{ Communication and collaboration } \\
\hline In the last week I have been able to communicate effectively with my baby's healthcare team & 108 & 4.250 (4.053 to 4.448$)$ & 4.407 (4.190 to 4.624$)$ & 0.05 \\
\hline $\begin{array}{l}\text { In the last week I have collaborated with my baby's healthcare team in the planning of care } \\
\text { for my baby }\end{array}$ & 107 & 3.843 (3.549 to 4.137$)$ & 3.426 (3.126 to 3.726$)$ & 0.02 \\
\hline In the last week I have been able to ask the healthcare team questions about my baby's care & 108 & $4.642(4.458$ to 4.827$)$ & $4.259(4.072-4.445)$ & 0.004 \\
\hline \multicolumn{5}{|l|}{ Privacy and confidentiality } \\
\hline In the last week the privacy of my baby's care was always considered and upheld & 107 & 4.435 (4.242 to 4.628$)$ & 4.322 (4.128 to 4.515$)$ & 0.33 \\
\hline In the last week the confidentiality of my baby's care was always considered and upheld & 108 & 4.474 (4.281 to 4.668$)$ & 4.281 (4.086 to 4.477$)$ & 0.08 \\
\hline In the last week I have overheard information about other babies & 107 & 2.196 (1.823 to 2.569$)$ & $2.683(2.281$ to 3.085$)$ & 0.46 \\
\hline
\end{tabular}


Table 3 Parental Stressor Scale for hospitalised infant for parents who completed PPCBR study for the whole trial cohort and stratified by the gestation of their infant (born $\leq 30$ or $>30$ weeks gestation)

\begin{tabular}{|c|c|c|c|c|}
\hline & \multirow{2}{*}{$\begin{array}{l}\text { Number of responses } \\
\text { analysed } \\
(n=126)^{*}\end{array}$} & \multicolumn{2}{|c|}{ Adjusted mean $(95 \% \mathrm{Cl})$} & \multirow[b]{2}{*}{ p Value } \\
\hline & & PPCBR & Non-PPCBR & \\
\hline \multicolumn{5}{|c|}{ All parents regardless of the gestation of their infants } \\
\hline Parental role & 105 & 3.477 (3.262 to 3.692 ) & 3.638 (3.421 to 3.856 ) & 0.09 \\
\hline Infant appearance & 107 & 4.210 (4.017 to 4.403$)$ & 4.087 (3.893 to 4.280$)$ & 0.15 \\
\hline Sight and sound & 102 & 2.327 (2.081 to 2.573 ) & 2.445 (2.197 to 2.693 ) & 0.34 \\
\hline Total score & 99 & 3.439 (3.268 to 3.609 ) & 3.471 (3.301 to 3.642 ) & 0.62 \\
\hline \multicolumn{5}{|c|}{ Parents of infants born $\leq 30$-week gestation } \\
\hline Parental role & 41 & 3.631 (3.255 to 4.007 ) & 3.443 (3.061 to 3.825 ) & 0.18 \\
\hline Infant appearance & 41 & 4.046 (3.710 to 4.383 ) & 4.145 (3.801 to 4.489 ) & 0.31 \\
\hline Sight and sound & 39 & $2.646(2.217$ to 3.076$)$ & 2.508 (2.059 to 2.957$)$ & 0.43 \\
\hline Total score & 37 & 3.505 (3.160 to 3.850$)$ & 3.444 (3.088 to 3.800$)$ & 0.57 \\
\hline \multicolumn{5}{|c|}{ Parents of infants born $>30$-week gestation } \\
\hline Parental role & 64 & 3.632 (3.355 to 3.909$)$ & 3.506 (3.240 to 3.773 ) & 0.33 \\
\hline Infant appearance & 66 & 4.108 (3.859 to 4.358 ) & 4.247 (4.007 to 4.486$)$ & 0.25 \\
\hline Sight and sound & 63 & 2.312 (1.999 to 2.625$)$ & 2.240 (1.946 to 2.534$)$ & 0.67 \\
\hline Total score & 62 & 3.442 (3.253 to 3.631$)$ & 3.439 (3.257 to 3.622 ) & 0.97 \\
\hline
\end{tabular}

Table 4 Characteristics and opinion of healthcare providers who participated on PPCBR study on knowledge, communication, privacy and impact on education of healthcare professionals'

\begin{tabular}{lc} 
& $\begin{array}{c}\text { Number (\%) or } \\
\text { median (IQR) (n=39) }\end{array}$ \\
\hline Participants demographics & 33 (85) \\
Female gender & $6.0(3-15)$ \\
Median years in work force (IQR) & 31 (79) \\
Nursing and midwifery profession & 8 (20) \\
Medical profession & 32 (82) \\
Knowledge, communication and collaboration* & 33 (85) \\
PPCBR improves parental knowledge and understanding about their baby's care? & 28 (72) \\
PPCBR facilitates effective communication between parents and the healthcare team? & 36 (92) \\
I spend less time explaining patient's condition and plan of care to families when families are present during rounds & 2 (5) \\
PPCBR encourages collaboration in the planning for the baby's future care? & 15 (38) \\
PPCBR creates more stress for the family & 21 (54) \\
Privacy, confidentiality and impact on healthcare providers education* & 16 (41) \\
PPCBR compromises privacy and confidentiality of babies in the NICU & 10 (26) \\
Families overhear information about other babies during clinical bedside rounds & 10 (26) \\
Parental presence significantly lengthened the duration of bedside rounds & 35 (90) \\
Parental presence at rounds significantly reduced teaching opportunities for the healthcare team & \\
PPCBR creates more stress for the healthcare providers & \\
Overall opinion* & \\
Do you agree parents should be able to attend clinical bedside rounds? &
\end{tabular}

participate in rounds. That is said, care must be taken to uphold confidentiality and to ensure that the discussion of potentially sensitive topics is not lost and that education of junior HCPs is not compromised.

Acknowledgements The authors are grateful to the parents and healthcare providers who took time to participate in this study. We also thank the following team members for their help with reviewing the study protocol and recruiting study participants: Jerome Poblete (BN, RN), Anne Cashel (ward clerk) and Dr Zsuzsoka
Kecskes (neonatologist). The authors acknowledge the statistical advice of Dr Bruce Shadbolt. We are also grateful for the Private Practice Fund, Canberra Hospital for their partial financial support of the study.

Contributors MEA-L was the chief investigator for the trial and the grant holder with Canberra Hospital Private Practice Fund. He chaired the trial management group, conceptualised and designed the trial. He contributed to design of interview questions, supervised data collection and analysed and contributed to data interpretation. He wrote the first draft of the paper and approved the final version of the manuscript. MB was a member of the trial management committee, supervised 
data collection and contributed to analysis and data interpretation. She contributed to data interpretation. She approved the final version of the manuscript. DB and JS were members of the trial management committee. They assessed parents for eligibility for the trial, recruited and collected and cleaned the data, contributed to data interpretation and approved the final version of the manuscript. DD contributed to the design of the interview questions, conducted the focus group discussion, carried out the qualitative analyses and drafted the qualitative result section. She critically reviewed the manuscript and approved the final manuscript as submitted.

Funding This study was funded, in part, by a grant from The Canberra Hospital Private Practice Fund, Canberra, Australia.

Competing interests None.

Ethics approval ACT Health Research Ethics Committee (ETH.6.11.121).

Provenance and peer review Not commissioned; externally peer reviewed.

Open Access This is an Open Access article distributed in accordance with the Creative Commons Attribution Non Commercial (CC BY-NC 4.0) license, which permits others to distribute, remix, adapt, build upon this work non-commercially, and license their derivative works on different terms, provided the original work is properly cited and the use is non-commercial. See: http://creativecommons.org/ licenses/by-nc/4.0/

\section{REFERENCES}

1 Gustafson K, Andrews T, Boyce M, et al. Parent presence on rounds in the neonatal intensive care unit (NICU) [Abstract]. In: Schell D, Kendrick T, Shekerdemian L, et al., eds. Six World Congress on Pediatric Critical Care Medicine Book of Abstracts. Volume 12. Sydney: Pediatric Critical Care Medicine, 2011:A22.

2 Cameron $\mathrm{MA}$, Schleien $\mathrm{CL}$, Morris MC. Parental presence on pediatric intensive care unit rounds. J Pediatr 2009;155:522-8.

3 Muething SE, Kotagal UR, Schoettker PJ, et al. Family centred bedside rounds: A new approach to patient care and teaching. Pediatrics 2007;119:829-32.

4 Bramwell R, Weindling M; FVWR Research Team. Families' views on ward rounds in neonatal units. Arch Dis Child Fetal Neonatal Ed 2005;90:F429-31.
5 Davidson JE, Powers K, Hedayat KM, et al. Clinical practice guidelines for support of the family in the patient-centred intensive care unit: American College of Critical Care Medicine task force 2004-2005. Crit Care Med 2007;35:605-22.

6 Phipps LM, Bartke CN, Spear DA, et al. Assessment of parental presence during bedside pediatric intensive care unit rounds: effect on duration, teaching and privacy. Pediatr Crit Care Med 2007;8:220-4.

7 Aronson PL, Yau J, Helfaer MA, et al. Impact of family presence during pediatric intensive care unit rounds on the family and medical team. Pediatrics 2009; 124:1119-25.

8 Lewis C, Knopf D, Chastain-Lorber K, et al. Patient, parent, and physician perspectives on pediatric oncology rounds. J Pediatr 1988;112:378-84.

9 Lehmann LS, Brancati FL, Chen MC, et al. The effect of bedside case presentations on patients' perceptions of their medical care. N Engl J Med 1997;336:1150-5.

10 Jackson K, Ternestedt BM, Schollin J. From alienation to familiarity: experiences of mothers and fathers of preterm infants. J Adv Nurs 2003;43:120-9.

11 Miles MS, Brunssen S. Parental stressor scale: infant hospitalization. Unpublished instrument manual, 1998. [cited 5 Oct 2013]. http://wwwuncedu/ mmiles/

12 Miles MS, Funk SG, Carlson J. Parental stressor scale: neonatal intensive care unit. Nurs Res 1993:42:148-52.

13 Perneger TV. What's wrong with Bonferroni adjustments? BMJ 1998;316:1236-8.

14 Sandelowski M. Focus on research methods-whatever happened to qualitative description? Res Nurs Health 2000;23:334-40

15 Giorgi A. Description versus interpretation-competing alternative strategies for qualitative research. J Phenomenol Psychol 1992;23:119-35.

16 Landry M, Lafrenaye S, Roy MC, et al. A randomized,controlled trial of bedside versus conference-room case presentation in apediatric intensive care unit. Pediatrics 2007;120:275-80.

17 Kassity N, Lockridge T. Should parents participate in patient rounds in the NICU? MCN Am J Matern Child Nurs 1999;24:64-5.

18 Watson D. Correcting for acquiescent response bias in the absence of a balanced scale-an application to class-consciousness. Sociol Methods Res 1992;21:52-88.

19 Leary M. Behavioral research methods. California: Wadsworth, 1991.

20 Sackett DL. Bias in analytic research. J Chronic Dis 1979;32:51-63. 